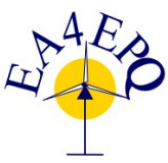

International Conference on Renewable Energies and Power Quality

(ICREPQ'13)

Bilbao (Spain), $20^{\text {th }}$ to $22^{\text {th }}$ March, 2013

Renewable Energy and Pourer Qualily. Fournal (RE\&PQJ)

ISSN 2172-038 X, No.11, March 2013

\title{
Comparison of the Harmonic Distortion of Current Source and Voltage Source Inverters
}

\author{
S. König, G. Herold ${ }^{1}$ \\ ${ }^{1}$ Chair of Electrical Energy Systems \\ University of Erlangen-Nuremberg \\ 91058 Erlangen (Germany) \\ Phone number: +49 9131 85-29526, e-mail: koenig@eev.eei.uni-erlangen.de
}

\begin{abstract}
This paper indicates a basic approach to compare the harmonic impact of current source and voltage source inverters. The latter as a kind of status quo for distributed generation and the first as an uncommon topology in the area of self-commutated infeed. Therefore it is shown how to calculate the fundamental frequency power exchange - what we want. Also the differences in the frequency dependant damping of the inverters higher harmonic output shall be analysed - what we do not want.
\end{abstract}

\section{Key words}

Current Source Inverter, Harmonics, Power Quality.

\section{Introduction}

EN 50160 [1] defines critical limits for several voltage quality aspects in electrical power grids. These specifications include maximum values for the harmonic content of the network voltages. In inverter technology it is always a chief goal to be able to maintain these parameters. Therefore one has a number of possibilities. One very basically decision is the type of inverter technology [2]. The main difference is the link type, whereas the voltage source inverter (VSI) contains a capacitor, the current source inverter (CSI) makes use of an inductor.

\section{Basic Differences between CSI and VSI}

In the following the two topologies shall be discussed, using the theory of the space phasor and the zero sequence. These two quantities transform the behaviour of the three phase system in one complex (1) and one real (2) component [3].

$$
\begin{aligned}
& \underline{v}=\frac{2}{3}\left(v_{R}+\underline{a} v_{S}+\underline{a}^{2} v_{T}\right) \quad \text { with } \quad \underline{a}=\mathrm{e}^{\mathrm{j} \frac{2 \pi}{3}} \\
& v_{0}=\frac{1}{3}\left(v_{R}+v_{S}+v_{T}\right)
\end{aligned}
$$

With that approach it is more demonstrative to illustrate the three phase networks. In their structure they resemble one phase networks but contain the time dependent information of all three phase quantities. It gets possible to analyse unbalances or transients in a way comparable to the symmetrical components.

Fig. 1 shows the basic space phasor network for the power exchange between the grid and a VSI. The power electronic part, including the source, the link element, the power semiconductors and the time-dependent switching functions [4] is represented by the time dependent voltage space phasor $\underline{u}_{V S I}$. Furthermore it is sometimes an advantage or necessary to add a filter inductance $L_{F}$. The grid side is represented by the network parameters $R_{G}, L_{G}$ and the grid voltage space phasor $\underline{u}_{G}$.

The VSI connects all three AC phases with the DC side and therefore generates a zero sequence voltage at its terminals. But if the reference potentials of the $\mathrm{AC}$ and the DC side are not connected, no zero sequence current can flow. As a consequence one does not need to consider the zero sequence network for normal operation conditions.

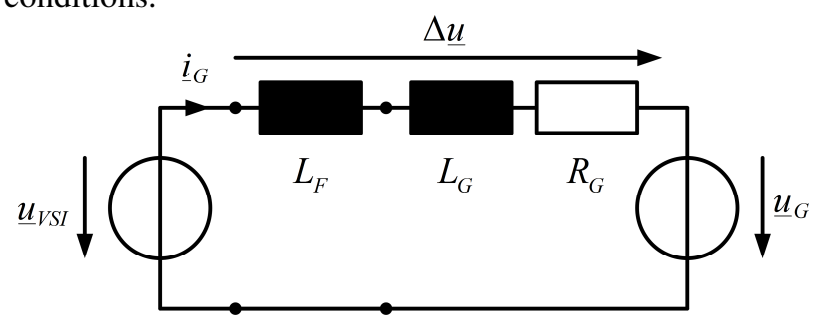

Fig. 1. Space phasor network of a VSI

The main difference between these two voltage sources is that the grid voltage can be supposed to be ideal sinusoidal, whereas the VSI source contains harmonic distortion.

Regarding the CSI, the grid part remains the same. But now the inverter is represented by a current space phasor, see Fig. 2. Besides, the previously optional filter element is always necessary in the form of a capacitance.

In contrast to the VSI the CSI does only connect two of the three AC phases with the DC side. The resulting zero sequence of the inducted current is zero and therefore there is again no need to regard a zero sequence network. 


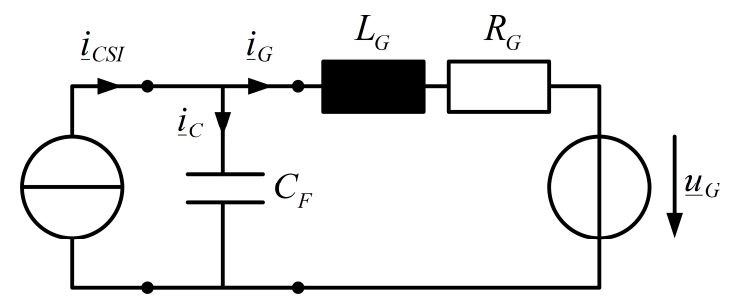

Fig. 2. Space phasor network of a CSI

\section{A. Fundamental Frequency Power Transmission VSI}

The goal of this part is to obtain equations for both inverters which show the fundamental frequency power exchange as a function of the network parameters and the sources.

Therefore it is possible to use the positive sequence of the symmetrical components. They are only able to represent mono-frequent sinusoidal stationary and symmetrical three phase systems and are thus a special case of space phasor and zero sequence. So, one can illustrate the dependencies in a vector diagram. This can be seen in the arbitrary example in Fig. 3, where $\delta$ is the so called transmission angle.

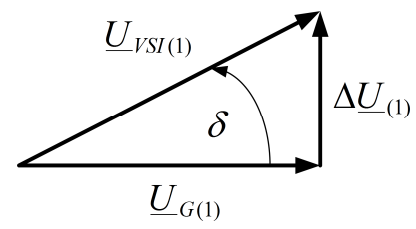

Fig. 3. Fundamental frequency vector diagram of the VSI

To get the exchanged power equations (3) and (4) are defined.

$$
\begin{gathered}
\underline{Z}_{F}+\underline{Z}_{G}=R_{G}+\mathrm{j} \omega\left(L_{F}+L_{G}\right)=Z \mathrm{e}^{\mathrm{j} \varphi_{Z}} \\
\underline{U}_{G(1)}=\frac{1}{k} \underline{U}_{V S I(1)} \mathrm{e}^{-\mathrm{j} \delta}
\end{gathered}
$$

Relation (4) indicates the theoretical possibility of a selfcommuted VSI, to arbitrarily vary the amplitude and the phase angle of the inverters output voltage in reference to the grid voltage.

Equation (5) shows the resulting fundamental frequency apparent power of a VSI at the point of common coupling.

$$
\underline{S}_{G(1)}=3 \underline{U}_{G(1)} \underline{I}_{G(1)}^{*}=3 \frac{U_{G(1)}^{2}}{Z} \mathrm{e}^{\mathrm{j} \varphi_{Z}}\left(k \mathrm{e}^{-\mathrm{j} \delta}-1\right)
$$

\section{B. Fundamental Frequency Power Transmission CSI}

For the CSI one must consider the current sources and the voltage sources contribution to the overall power exchange. Therefore it is necessary to calculate the voltage at the capacitance as a function of these two sources with Thévenin's theorem. The equations (6) - (8) indicate summarized impedances to reduce the equations.

$$
\begin{aligned}
& \underline{Z}_{P}=\frac{1}{\mathrm{j} \omega C_{F}+\frac{1}{R_{G}+\mathrm{j} \omega L_{G}}} \\
& \underline{Z}_{G}=R_{G}+\mathrm{j} \omega L_{G} \\
& \underline{Z}_{S}=R_{G}+\mathrm{j} \omega L_{G}+\frac{1}{\mathrm{j} \omega C_{F}}
\end{aligned}
$$

With the capacitors impedance $\underline{Z}_{C}=\frac{1}{\mathrm{j} \omega C_{F}}$ one obtains (9) for the voltage contribution of the current source.

$$
\underline{U}_{C(1) I}=\underline{I}_{C S I(1)}\left(1-\frac{\underline{Z}_{P}}{\underline{Z}_{G}}\right) \underline{Z}_{C}
$$

The portion of the voltage source is

$$
\underline{U}_{C(1) I I}=\underline{U}_{G(1)} \underline{\underline{Z}}_{C}
$$

As a result the overall fundamental frequency power for the CSI at the point of common coupling is

$$
\begin{aligned}
& \underline{S}_{G(1)}=3 \underline{U}_{G(1)} \underline{I}_{G(1)}^{*}= \\
& =3 \underline{U}_{G(1)}(\underline{I}_{C S I(1)}-\underbrace{\frac{1}{\underline{Z}_{C}}\left(\underline{U}_{C(1) I}+\underline{U}_{C(1) I I}\right)}_{\underline{I}_{C(1)}})^{*}= \\
& =3 \underline{U}_{G(1)}\left(\underline{I}_{C S I(1)} \underline{\underline{Z}}_{P} \underline{Z}_{G}-\underline{U}_{G(1)} \frac{1}{\underline{Z}_{S}}\right)^{*}
\end{aligned}
$$

Now it is possible to calculate the expected fundamental frequency power for both inverter types with (5) and (11) as a function of the network- and the source- parameters.

\section{Bode Plot of the VSI}

Both networks in Fig. 1 and Fig. 2 have a different influence on the harmonic content of the grid current. To be able to evaluate the transmission of the sources harmonic content to the point of common coupling both transmission functions shall be determined.

For the VSI in Fig. 1 the input variable is the voltage difference $\Delta \underline{U}(\omega)$ between the distorted inverter voltage and the grid voltage, the output parameter is the grid current $\underline{I}_{G}(\omega)$. Both are functions of the angular frequency $\omega=2 \pi f$, where $f$ is the frequency.

Because the grid source is estimated to contain only the fundamental frequency, it will only have an impact on the grid current at the fundamental angular frequency $\omega_{1}=2 \pi 50 \mathrm{~Hz}$.

The resulting voltage drop at the longitudinal impedance is given in equation (12).

$$
\Delta \underline{U}(\omega)=\left\{\begin{array}{cc}
\underline{U}_{V S I}(\omega)-\underline{U}_{G}(\omega) & \text { for } \omega=\omega_{1} \\
\underline{U}_{V S I}(\omega) & \text { else }
\end{array}\right.
$$

Using complex phasor representation the transmission function $\underline{G}_{V S I}(\omega)$ of Fig. 1 is given by (13) for $\omega \neq \omega_{1}$.

$$
\underline{G}_{V S I}(\omega)=\frac{\underline{I}_{G}(\omega)}{\Delta \underline{U}(\omega)}=\frac{1}{\mathrm{j} \omega\left(L_{G}+L_{F}\right)+R_{G}}=\frac{1 / R_{G}}{\mathrm{j} \frac{\omega}{\omega_{0}}+1}
$$

Fig. 4 shows the corresponding bode plot. It can be seen that for frequencies smaller than the cutoff frequency $f_{0}$

$$
f_{0}=\frac{\omega_{0}}{2 \pi}=\frac{R_{G}}{2 \pi\left(L_{G}+L_{F}\right)}
$$

the transmission functions effect is constant and that for higher frequencies a damping of $20 \mathrm{~dB}$ per decade occurs. 


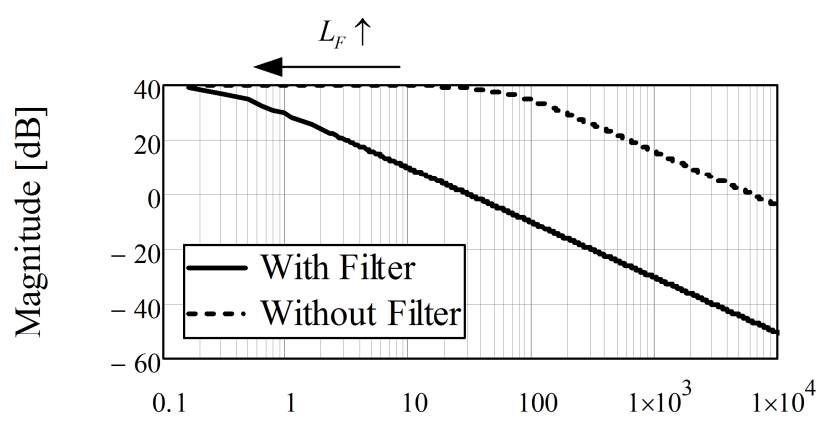

Frequency $[\mathrm{Hz}]$

Fig. 4. Bode plot of the VSI

As a further consequence it can be seen, that the use of an additional filter inductance reduces the cutoff frequency and leads to a higher damping for the higher frequency range.

\section{Bode Plot of the CSI}

As for the VSI the grid voltage only affects the fundamental frequency parts. For example the grid current is now given by relation (15).

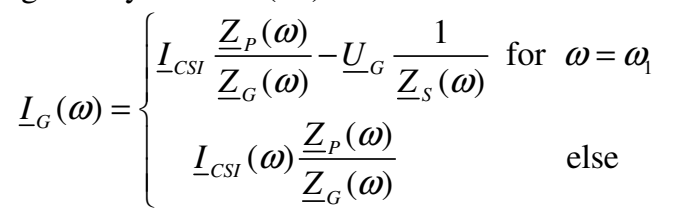

For $\omega \neq \omega_{1}$ the transmission function is

$$
\begin{aligned}
\underline{G}_{C S I}(\omega) & =\frac{\underline{I}_{G}(\omega)}{\underline{I}_{C S I}(\omega)}=\frac{\underline{Z}_{P}}{\underline{Z}_{G}} \\
& =\frac{1}{(\mathrm{j} \omega)^{2} L_{G} C_{F}+\mathrm{j} \omega R_{G} C_{F}+1} \\
& =\omega_{0}^{2} \frac{1}{(\mathrm{j} \omega)^{2}+(\mathrm{j} \omega) 2 d \omega_{0}+\omega_{0}^{2}}
\end{aligned}
$$

It does not take into account the grid voltages contribution to the grid current. Now the cutoff frequency is

$$
f_{0}=\frac{\omega_{0}}{2 \pi}=\frac{1}{2 \pi \sqrt{L_{G} C_{F}}}
$$

And the damping factor $d$ is

$$
d=\frac{R_{G}}{2} \sqrt{\frac{C_{F}}{L_{G}}}
$$

Fig. 5 shows the resulting bode plot of the CSI network. In contrast to the VSI transmission network there are two main differences. The first one is the (network parameter dependent) resonance area around the cutoff frequency in the plot. That means that a stimulation of the network with input currents of a frequency in this area leads to an output current that is higher than the input.

The second difference is the higher damping of $40 \mathrm{~dB}$ per decade for the frequencies above the cutoff frequency.

With an increasing filter capacitance it can be seen in equation (17), that the cutoff frequency decreases again and the damping factor $d$ increases, c.f. (18) (which leads to a lower resonance rise).

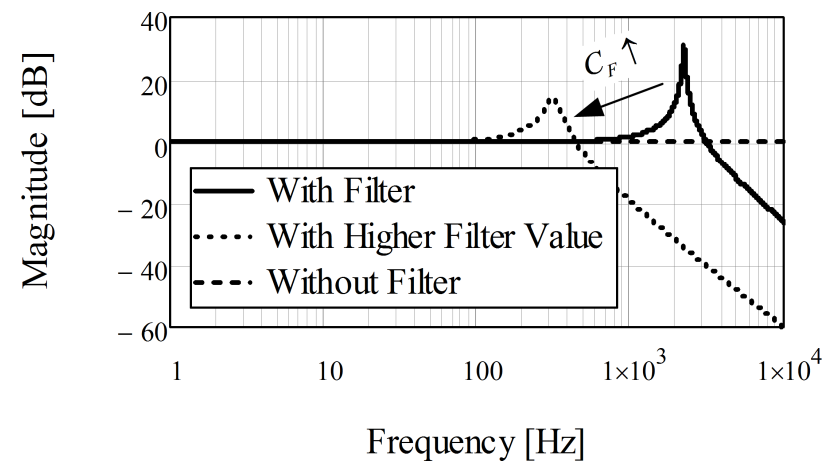

Fig. 5. Bode plot of the CSI

\section{Comparison of VSI and CSI}

Now, the consequences of this different behaviour shall be outlined. For this comparison two conditions were defined. First of all, of course both inverter topologies shall feed in the same amount of fundamental apparent power. So for both cases

$$
\underline{S}_{G(1)}=3 \underline{U}_{G(1)} \underline{I}_{G(1)}^{*}
$$

has to be indicated.

This leads to a demanded fundamental output voltage of the VSI of

$$
\underline{U}_{V S I(1)}=\underline{I}_{G(1)}\left(\underline{Z}_{G}+\underline{Z}_{F}\right)+\underline{U}_{G(1)}
$$

And the following fundamental output current for the CSI

$$
\underline{I}_{C S I(1)}=\left(\underline{I}_{G(1)}+\underline{U}_{G(1)} \frac{1}{\underline{Z}_{S}}\right) \underline{\underline{Z}}_{G}
$$

The second condition was the assumption of an equally distorted output spectrum for both inverters. Because this paper shows the principle differences a simple fundamental frequency switching function, that also contains lower harmonic distortion, was chosen. The resulting space phasor spectrum $v(n)$, where $n$ is the harmonic order, is given with (22) and can be seen in Fig. 6 [5].

$$
\begin{aligned}
& c(n)=\left(1+\mathrm{e}^{\mathrm{j}(n-1) \pi}\right)\left(1+\underline{\mathrm{a}}^{2 n+1}+\underline{\mathrm{a}}^{n+2}\right) \\
& v(n)=c(n) \frac{1}{\pi} \frac{\left(\mathrm{j}^{2}\right)^{n}}{n} \sin \left(n \frac{\pi}{6}\right)
\end{aligned}
$$

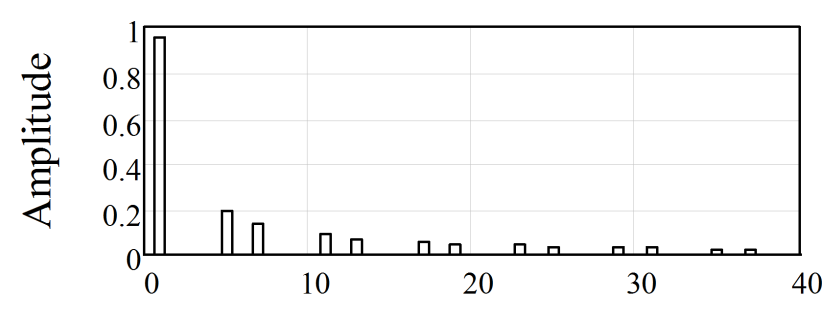

Harmonic Order

Fig. 6. Referred output spectrum

The demanded output values and this spectrum lead to the necessary DC voltage of the VSI and the required DC current of the CSI.

Fig. 7 shows the results for the CSI. As can be seen the fundamental frequency is reduced through the grid 


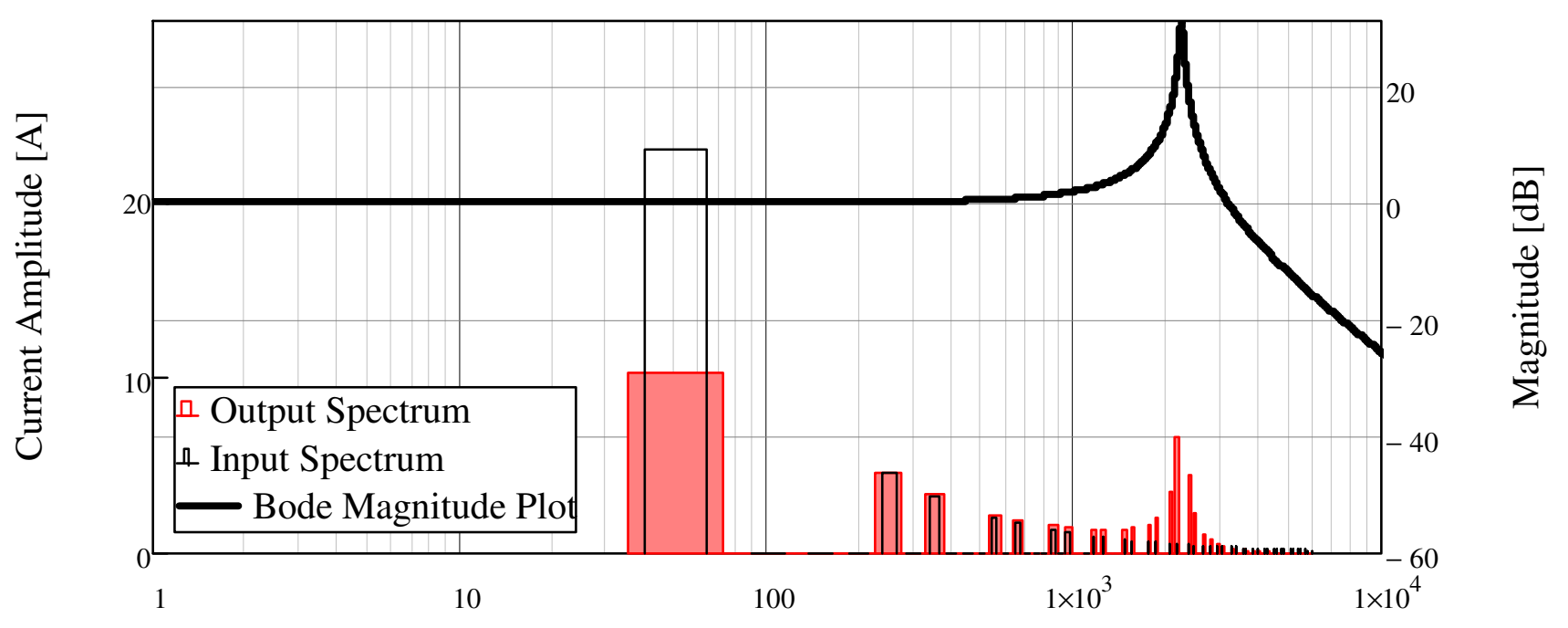

Frequency $[\mathrm{Hz}]$

Fig. 7. CSI spectra of inverter- and grid- current

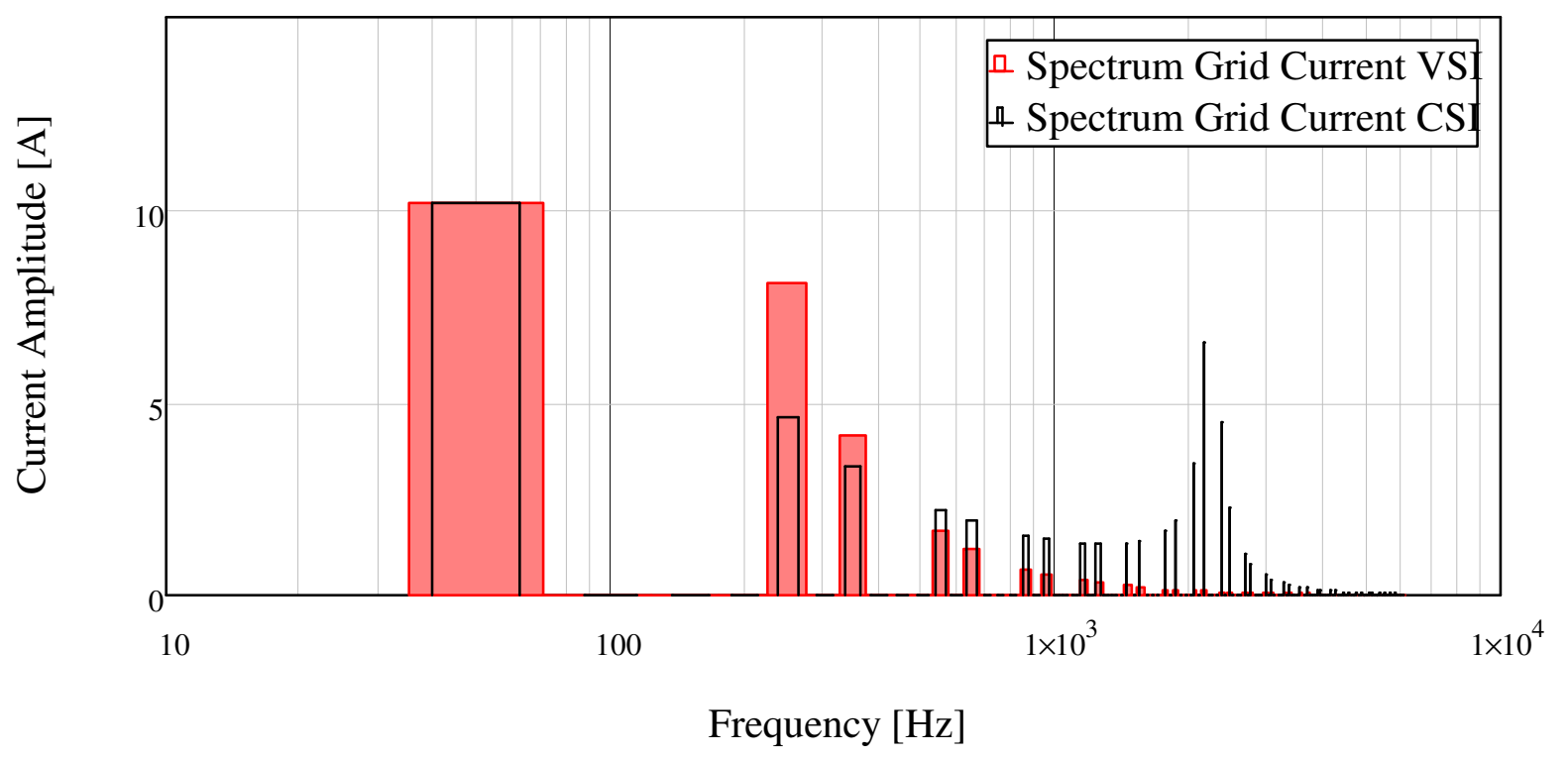

Fig. 8. Comparison of VSI and CSI grid current spectrum

voltage and not because of the transmission function. Furthermore the frequencies in the area of the cutoff frequency are boosted following the transmission function. For frequencies higher than the cutoff frequency the output spectrum is damped and nearly not visible anymore.

Now of course the question arises, how the VSI spectrum looks like. It is shown in comparison to the CSI spectrum in Fig. 8. This figure indicates that there is no resonance area and the higher the harmonic order, the higher the damping, c.f. transmission function in Fig. 4.

\section{Conclusion}

The CSI network offers a higher damping for frequencies above the cutoff frequency, but one has to handle the resonance zone. Those are in the same instance the advantage and the disadvantage of the VSI network: no resonance, but lower damping. It must be said, that the concrete results are dependent on the network parameters and the chosen switching functions of the inverters.

\section{References}

[1] Voltage characteristics of electricity supplied by public distribution networks; (German version), DIN EN 50160, 2011-02-00.

[2] M. Bierhoff, Pulse Width Modulation for Current Source Converters - A Detailed Concept. Available: http://www.tf.uni-kiel.de/etit/LEA/dlopen/IECON06_Bierhoff.pdf (2011, Oct. 04).

[3] G. Herold, Electrical Power Supply I (German): Threephase systems, powers, economy, 2nd ed. Wilburgstetten: J. Schlembach Fachverlag, 2005.

[4] G. Holmes and T. A. Lipo, Pulse width modulation for power converters: Principles and practice. New York ; Chichester: Wiley, 2002.

[5] G. Herold, Electrical Power Supply V (German): Converters in three-phase systems, $1 \mathrm{st}$ ed. Wilburgstetten: J. Schlembach Fachverlag, 2009. 